\title{
Tipos histológicos e mortalidade por câncer gástrico em São Paulo
}

\author{
Hystological types and mortality for gastric cancer \\ in São Paulo, Brazil
}

Carlos Marigo 1

Mario H. Okuyama 2

Giulio Cesare Santo 2

\footnotetext{
1 Fundação Oncocentro de São Paulo. Rua Oscar Freire 2396, São Paulo, SP 05409-012, Brasil.

Internet:

http://eu.ansp.br:80/ fosp. E.mail:

fundonco@fesesp.org.br. E.mail (via FAPESP):

fosp@eu.ansp.br

2 Faculdade de Ciências Médicas, Santa Casa de São

Paulo. Rua Cesário Mota

Junior 112, São Paulo, SP 01221-900, Brasil.
}

Abstract The objective of this study was to analyze, according to Lauren's classification, the prevalence of a hystological type - intestinal or diffuse - , among gastric carcinomas. The authors reviewed 650 hystologi cal sections from a Hospital in the City of São Paulo during a 30year period, foll owing the definitions of the above classification. After the 50's the intestinal type proved to be prevalent, reaching $62.74 \%$ of all cases of gastric cancer in the 80 's, and showing a ratio of intestinal/diffuse type of 1.71. Other large series of cases whose diagnoses foll owed the nomenclature of WHO Hystol ogical Classification showed to be inadequate for this study. The prevalence of the intesti nal type of gastric carcinoma seems to disagree with the decreasing rates of mortality due to this disease in the State of São Paulo, in the same period. Further studies, such as the analysis of incidence rates and prevalence of Helicobacter pylori, among others, are necessary for a better understanding of these data.

Key words Gastric Cancer; Stomach; Hystologic Types; Mortality; TimeTrend

Resumo A intenção dos autores é verificar qual tipo histológico prevalece, se o intestinal ou difuso, segundo a classi fi cação de Lauren. Os preparados histopatológi cos de 650 casos de um hospital da cidade deSão Paulo, cobrindo um período de trinta anos, foram revistos, aplicando-se as defini ções da classi fi cação aci ma menci onada. Os resultados mostraram que o tipo intestinal tornou-se prevalente após a década de 50, atingindo a cifra de 62,74\% entre todos os casos de carci noma na década de 80, sendo de 1,71 a razão intesti nal/di fuso. Outras séries de casos cuj os diagnósticos segui ram a nomenclatura da Classificação Histológica da WHO não se mostraram adequadas para este tipo de estudo. A prevalência do tipo intestinal contrasta, aparentemente, com o decrésci mo das taxas de mortal idade por esta doença no Estado de São Paulo no mesmo período. São necessários estudos futuros, incluindo análises de taxas de inci dência e prevalência do Helicobacter pylori, entre outros, para mel hor interpretação desses dados.

Palavras-chave Câncer Gástrico; Estômago; Tipos Histológi cos; Mortalidade; Tendência Temporal 
Introdução

A necessidade da caracterização precisa do tipo histológico dos carcinomas gástricos devese à existência de uma classificação histológica de conotação epidemiológica. Essa classificação foi baseada em achados microscópicos relatados por Järvi \& Lauren (1951), sendo que Lauren (1965) consolidou a referida classificação. Esses autores propõem a separação de dois grandes grupos que constituem a maioria dos casos: o tipo denominado intestinal (epidêmico) e o tipo difuso (endêmico) havendo, além disso, os casos não característicos. TrabaIhos posteriores de Muñoz et al. (1968) e Correa et al. (1973) demonstram que o tipo intestinal incide muito mais nas áreas geográficas de alto risco do que nas de baixo risco, ao passo que o tipo difuso não revela diferenças significantes.

Verificou-se ainda que o decréscimo da mortalidade e da inci dência ocorrida em vários países deveu-se à diminuição marcante do tipo intestinal sem afetar o tipo difuso, como demonstraram Muñoz \& Asvall (1971), Hanai et al. (1982) e Jonasson et al. (1994).

O presente trabalho tem o objetivo de iniciar esses estudos na Fundação Oncocentro de São Paulo (FOSP).

Tabela 1

Número de casos e relação intestinal/difuso.

\begin{tabular}{lcccc}
\hline Tipo histológico & \multicolumn{4}{c}{ Anos } \\
& $1953-54$ & $1963-64$ & $1973-74$ & $1983-84$ \\
\hline Intestinal & 68 & 90 & 94 & 104 \\
Difuso & 85 & 66 & 58 & 61 \\
Relação I/D & 0,80 & 1,36 & 1,62 & 1,71 \\
\hline
\end{tabular}

Tabela 2

Mortalidade por câncer do estômago* no Estado de São Paulo, por sexo.

\begin{tabular}{lrrrrr}
\hline Sexo & \multicolumn{5}{c}{ Anos } \\
& 1970 & 1975 & 1980 & 1985 & 1992 \\
\hline Masculino & 35,7 & 30,8 & 27,7 & 23,4 & 20,3 \\
Feminino & 16,0 & 12,6 & 11,1 & 9,2 & 8,6 \\
\hline
\end{tabular}

* taxa por 100.000 habitantes, padronizada.
Material e métodos

Utilizou-se como fontes de diagnósticos histopatológicos o banco de dados do registro hospitalar de câncer da Fundação Oncocentro de São Paulo (FOSP, 1996) referentes ao ano de 1991, além dos diagnósticos existentes nas publicações de Brumini et al. (1982) referentes ao período 1976-1980 e do registro nacional de patologia tumoral (INC, 1991) referentes ao período 1981-1985. Essas informações constavam dos laudos anatomopatológicos, mas os preparados histológicos não foram revistos. Fez-se uso, portanto, apenas da nomenclatura do laudo para compor os dois grupos. A outra série de casos faz parte dos arquivos do Departamento de Patologia da Santa Casa de São PauIo, dos quais foram analisados todos os casos de dois anos em cada década, começando pelo biênio 1953-1954 até o biênio 1983-1984, num total de 650 casos. Deste material foram revistos todos os preparados histológicos, empregando-se para a classificação aqueles critérios propostos por Lauren (1965), retirando-se aqueles não classificáveis, que somaram 24 casos. Quando havia mais de um exame histopatológico, isto é, biópsias e espécime cirúrgico, apenas este último foi utilizado. Na ocorrência somente de preparados de biópsias, apenas foram incluídos no estudo aqueles cujo material era suficiente para permitir classificação. Obtidos esses dados e distribuídos apenas os pertinentes aos dois grupos (intestinal e difuso), calculou-se a freqüência relativa e a relação simples entre os dois tipos, aplicando-se como denominador o tipo difuso.

Para o estudo da mortalidade, foram utilizados os registros de certificado de óbitos armazenados pela Fundação de Sistema Estadual de Análises de Dados do Estado de São Paulo (Fundação SEADE) dos anos de 1970, 1975, 1980, 1985 e 1992, os quais já foram publicados em trabal hos colaborativos FOSP/SEADE (Fonseca, 1992; Fonseca \& Mameri, 1994).

\section{Resultados}

Quanto aos tipos histológicos, os índices obtidos da relação tipo intestinal/ tipo difuso referem-se a ambos os sexos e foram os seguintes:

Registro hospitalar da FOSP, 1991: 8,22

Registro nacional de patologia tumoral, 1976-80: 10,9; 1981-85: 8,44.

Departamento de Patologia da Santa Casa de São Paulo (Tabela 1).

Quanto à mortalidade, foram obtidas as seguintes taxas por cem mil habitantes (Tabela 2). 


\section{Comentários}

Os índices tipo intestinal/tipo difuso obtidos no Registro Hospitalar da FOSP, assim como no Registro de Patologia Tumoral revelaram-se muito altos, o que provavelmente não corresponde à realidade, pois índices com as magnitudes observadas neste trabalho não são observados mesmo nas regiões de maior incidência mundial. Isso mostra que não é possível extrapolar simplesmente os diagnósticos histológicos reportados nos laudos de rotina para a classificação de Lauren, pois esta tem nomenclatura própria e obedece critérios microscópios determinados.

Esse aspecto ficou patente com os resultados obtidos após revisão dos preparados histológicos de um hospital geral (Santa Casa de São Paulo). A revisão tinha por objetivo adequar os diagnósticos utilizando os critérios específicos da classificação "epidemiológica". Os índices obtidos da relação intestinal/difuso tornaramse muito menores, e revelaram uma "viragem" ao passarmos da década de 50 para a de 60. A partir desta última, ficaram sempre acima de um (predomínio do tipo intestinal) com tendência a aumentar progressivamente (Figura 1). Verifica-se na Figura 1 que o aumento do índice deve-se não só à crescente freqüência relativa do tipo intestinal (atingindo $62,64 \%$ dentre todos os carcinomas gástricos em 1983/84), como à queda da freqüência do tipo difuso a partir de 1953/54, este último com tendência a estabilizar (daí as denominações epidêmico e endêmico). Cumpre lembrar ainda que, desses pacientes admitidos na Santa Casa de São Paulo, cerca de $43 \%$ não nasceram no Estado de São Paulo, sendo oriundos principalmente de Minas Gerais e Nordeste o que poderá modificar a representatividade da amostra no que se refere a possíveis fatores de risco ambientais. Além disso, após a identificação do Heli cobacter pyl ori no estômago por Warren \& Marshal (1984), essa bactéria passou a ser freqüentemente relacionada com gastrite, úlcera e neoplasia gástrica, tornando-se portanto mais um fator a ser analisado.

Esses índices relativos seriam indicativos indiretos da estabilidade ou mesmo do possível aumento da incidência global de câncer gástrico, pois, como já vimos, nas áreas de alto risco predomina o tipo intestinal. Infelizmente as taxas de incidência conhecidas no estado referem-se apenas ao município da capital dos anos 1969, 1973 e 1978 (Mirra \& Franco, 1987), mas não contradizem aqueles indicativos histopatológicos. A ausência de dados após 1978 não permite melhores estudos da tendência
Figura 1

Freqüência relativa dos tipos intestinal e difuso e relação $I / D$, ambos os sexos, em trinta anos. Hospital Geral.

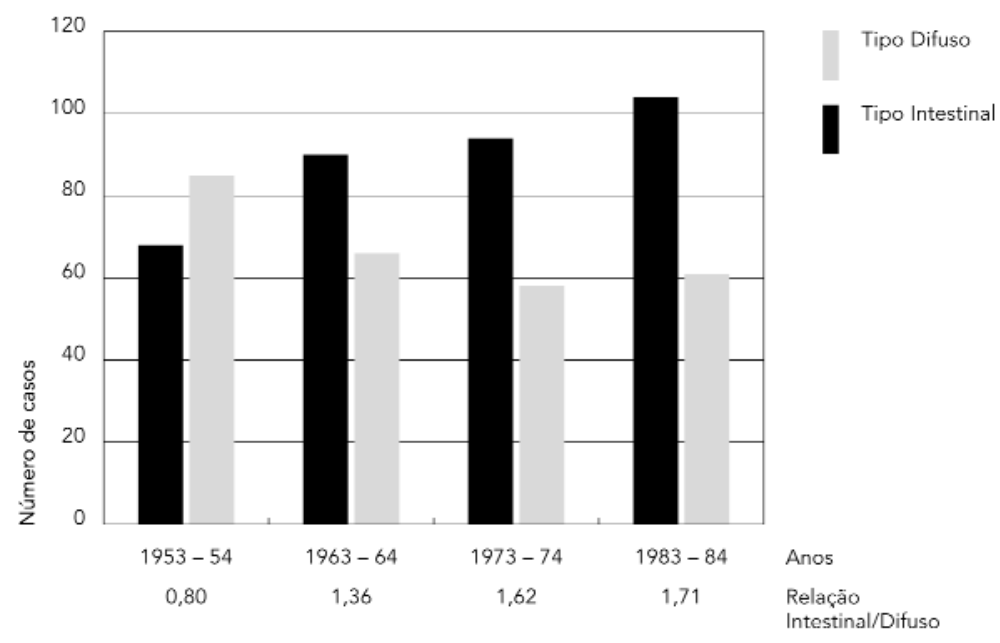

Fonte: Depto. de Patologia da Santa Casa de São Paulo.

Figura 2

Coeficiente de mortalidade por câncer de estômago no Estado de São Paulo, 19701992, segundo sexo*.

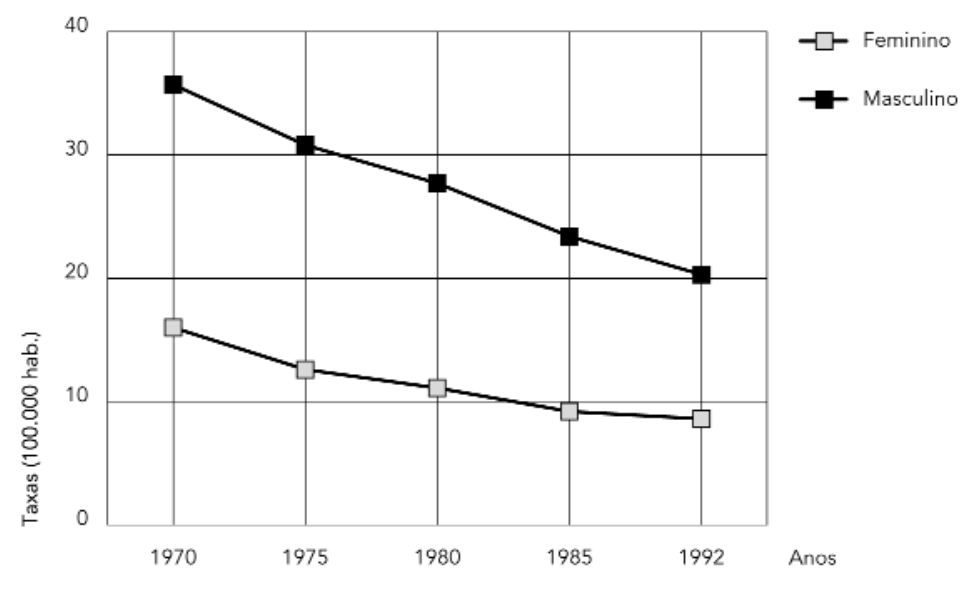

* Padronizados segundo população mundial.

Fonte:FOSP/SEADE (Fonseca \& Mameri).

temporal quanto à incidência.

Quanto à mortalidade, porém, não há dúvida que diminuiu no Estado nas últimas décadas (Figura 2). Essa queda está ocorrendo desde a década dos 50 em numerosos países, inclusive nos da América Latina, como pode ser visto nas publicações Aoki et al. (1992) e De Stefani et al. (1994), assim como nas Estatísti- 
cas de Mortalidade do Brasil (FNS, 1977, 1978, 1979, 1980, 1981, 1982, 1983, 1984, 1985, 1986, 1987, 1988, 1989, 1990, 1991, 1992, 1993).

Estaríamos, portanto, diante de fatos provavelmente conflitantes, quais sejam: redução da mortalidade por câncer gástrico e aumento da diferença proporcional do tipo intestinal. Tendo em vista que o número de diagnósticos de câncer precoce, fator que reduz a mortalidade, não é significante em nosso meio, o mais provável é que essa queda deva-se à diminui-

\section{Referências}

AOKI, K.; HAYAKAWA, N.; KURIHARA, M. \& SUZUKI, S., 1992. Death Rates for Malignant Neoplasms for Sel ected Sites by Sex and Five-Year Age Group in 33 Countries, 1953-57 to 1983-87. International Union Against Cancer - The University of Nagoya Coop. Press, Nagoya.

BRU MINI, R., 1982. Câncer no Brasil: dados histopatológi cos 1976/80. Campanha Nacional de Controle do Câncer, Ministério da Saúde, Rio de Janeiro.

CORREA, P.; SASANO, N.; STEMMERMANN, N. \& HAENSZEL, W., 1973. Pathology of gastric carcinoma in Japanese populations: comparisons between Miyagi prefecture, Japan an Hawaii. Journal of the National Cancer Institute, 51:1.449-459.

DE STEFANI, E.; FIERRO, L.; BARRIOS, E. \& RONCO, A., 1994. Cancer mortality trends in Uruguay 1953-1991. International Journal of Cancer, 56:634-639.

DOLL, R., 1991. Progress against cancer: an epidemiologic assessment. American Journal of Epidemiology, 134:675-688.

FNS (FUNDAÇÃO NACIONAL DE SAÚDE), 1977. Estatísticas de Mortalidade- Brasil. Brasília: Ministério da Saúde, Fundação Nacional de Saúde. Centro Nacional de Epidemiologia. Coordenação de Informações e Análise da Situação da Saúde.

FNS (FUNDAÇÃO NACIONAL DE SAÚDE), 1978. Estatísticas de Mortalidade - Brasil. Brasília: Ministério da Saúde, Fundação Nacional de Saúde. Centro Nacional de Epidemiologia. Coordenação de Informações e Análise da Situação da Saúde.

FNS (FUNDAÇÃO NACIONAL DE SAÚDE), 1979. Estatísticas de Mortalidade- Brasil. Brasília: Ministério da Saúde, Fundação Nacional de Saúde. Centro Nacional de Epidemiologia. Coordenação de Informações e Análise da Situação da Saúde. ção da incidência. Em países onde a incidência é conhecida, essa redução tem ocorrido sem intervenção programada, provavelmente à custa da melhor conservação dos alimentos e dieta rica em determinados nutrientes (Howson et al., 1986; Doll, 1991), mas o tipo histológico intestinal é que traduz essa redução.

Estudos futuros que, além de outros parâmetros, incluam dados temporais de incidência poderão esclarecer o que está acontecendo em nosso meio.
FNS (FUNDAÇÃO NACIONAL DE SAÚDE), 1980. Estatísticas de Mortalidade- Brasil. Brasília: Ministério da Saúde, Fundação Nacional de Saúde. Centro Nacional de Epidemiologia. Coordenação de Informações e Análise da Situação da Saúde.

FNS (FUNDAÇÃO NACIONAL DE SAÚDE), 1981. Estatísticas de Mortalidade- Brasil. Brasília: Ministério da Saúde, Fundação Nacional de Saúde. Centro Nacional de Epidemiologia. Coordenação de Informações e Análise da Situação da Saúde.

FNS (FUNDAÇÃO NACIONAL DE SAÚDE), 1982. Estatísticas de Mortalidade- Brasil. Brasília: Ministério da Saúde, Fundação Nacional de Saúde. Centro Nacional de Epidemiologia. Coordenação de Informações e Análise da Situação da Saúde.

FNS (FUNDAÇÃO NACIONAL DE SAÚDE), 1983. Estatísticas de Mortalidade - Brasil. Brasília: Ministério da Saúde, Fundação Nacional de Saúde. Centro Nacional de Epidemiologia. Coordenação de Informações e Análise da Situação da Saúde.

FNS (FUNDAÇÃO NACIONAL DE SAÚDE), 1984. Estatísticas de Mortalidade- Brasil. Brasília: Ministério da Saúde, Fundação Nacional de Saúde. Centro Nacional de Epidemiologia. Coordenação de Informações e Análise da Situação da Saúde.

FNS (FUNDAÇÃO NACIONAL DE SAÚDE), 1985. Estatísticas de Mortalidade - Brasil. Brasília: Ministério da Saúde, Fundação Nacional de Saúde. Centro Nacional de Epidemiologia. Coordenação de Informações e Análise da Situação da Saúde.

FNS (FUNDAÇÃO NACIONAL DE SAÚDE), 1986. Estatísticas de Mortalidade - Brasil. Brasília: Ministério da Saúde, Fundação Nacional de Saúde. Centro Nacional de Epidemiologia. Coordenação de Informações e Análise da Situação da Saúde. 
FNS (FUNDAÇÃO NACIONAL DE SAÚDE), 1987. Estatísticas de Mortalidade- Brasil. Brasília: Ministério da Saúde, Fundação Nacional de Saúde. Centro Nacional de Epidemiologia. Coordenação de Informações e Análise da Situação da Saúde.

FNS (FUNDAÇÃO NACIONAL DE SAÚDE), 1988. Estatísticas de Mortalidade - Brasil. Brasília: Ministério da Saúde, Fundação Nacional de Saúde. Centro Nacional de Epidemiologia. Coordenação de Informações e Análise da Situação da Saúde.

FNS (FUNDAÇÃO NACIONAL DE SAÚDE), 1989. Estatísticas de Mortalidade- Brasil. Brasília: Ministério da Saúde, Fundação Nacional de Saúde. Centro Nacional de Epidemiologia. Coordenação de Informações e Análise da Situação da Saúde.

FNS (FUNDAÇÃO NACIONAL DE SAÚDE), 1990. Estatísticas de Mortalidade- Brasil. Brasília: Ministério da Saúde, Fundação Nacional de Saúde. Centro Nacional de Epidemiologia. Coordenação de Informações e Análise da Situação da Saúde.

FNS (FUNDAÇÃO NACIONAL DE SAÚDE), 1991. Estatísticas de Mortalidade- Brasil. Brasília: Ministério da Saúde, Fundação Nacional de Saúde. Centro Nacional de Epidemiologia. Coordenação de Informações e Análise da Situação da Saúde.

FNS (FUNDAÇÃO NACIONAL DE SAÚDE), 1992. Estatísticas de Mortalidade- Brasil. Brasília: Ministério da Saúde, Fundação Nacional de Saúde. Centro Nacional de Epidemiologia. Coordenação de Informações e Análise da Situação da Saúde.

FNS (FUNDAÇÃO NACIONAL DE SAÚDE), 1993. Estatísticas de Mortalidade- Brasil. Brasília: Ministério da Saúde, Fundação Nacional de Saúde. Centro Nacional de Epidemiologia. Coordenação de Informações e Análise da Situação da Saúde.

FONSECA, L. A. M., 1992. Mortalidade por câncer no Estado de São Paulo. Oncologia Atual, 2:6-14.

FONSECA, L. A. M. \& MAMERI, C. P., 1994. Mortalidadepor Câncer no Estado deSão Paulo. São PauIo: SEADE/FOSP.

HANAI, A.; FUJIMOTO, I. \& TANIGUCHI, H., 1982. Trends of stomach cancer incidence and histological types in Osaka. In: Trends in Cancer Incidence: Causes and Pratical Implications (Magnus K., ed.), pp. 43-154, New York: Hemisphery Public Corp.
HOWSON, C. P.; HIYAM A, T. \& WYNDER, E. L., 1986. The decline in gastric cancer: epidemiology of an unplanned triumph. Epidemiologic Reviews, 8:127.

JÄRVI, O. \& LAUREN, P., 1951. On the role of heterotopias of the intestinal epithelium in the pathogenesis of gastric cancer. Acta Pathologica et Microbiologica Scandinavia, 29:26-44.

JONASSON, L.; HALLGRIMSSON, J.; SIGVALDASON, H.; OLAFSDOTTIR, G. \& TULINIUS, H. 1994. Gastric cancer in Iceland: a retrospective study of resected gastric specimens in a high-risk contry during 30 years (1960-1989). International Journal of Cancer, 57:793-798.

LAUREN, P., 1965. The two histological main types of gastric carcinoma: diffuse and so-called intestinal-type carcinoma: an attempt at a histoclinical classification. Acta Pathologica et Microbiologica Scandinavia, 64:31-49.

MIRRA, A. P. \& FRANCO, E. L., 1987. Cancer Mortality in São Paulo, Brazil. Vol. 3, São Paulo: Cancer Epidemiology Monograph Series. Ludwig Institute for Cancer Research.

MUÑOZ, N. \& ASVALL, J., 1971. Time trends of intestinal and diffuse types of gastric cancer in Norway. International Journal of Cancer, 8:144-157.

MUÑOZ, N.; CORREA, P.; CUELLO, C. \& DUQUE, E., 1968. Histologic types of gastric carcinoma in high-and low-risk areas. International Journal of Cancer, 3:809-818.

FOSP (FUNDAÇÃO ONCOCENTRO DE SÃO PAULO), 1996. Registro Hospitalar de Câncer. São Paulo: Unidade de Epidemiologia. Fundação Oncocentro de São Paulo.

INC (INSTITUTO NACIONAL DE CÂNCER), 1991. Registro Nacional de Patologia Tumoral. Diagnóstico de Câncer no Brasil -1981/85. Rio de Janeiro: Ministério da Saúde. Instituto Nacional de Câncer. Programa de Oncologia.

WARREN, J. R. \& MARSHALL, B. J., 1984. Unidentified curved bacilli in the stomach of pacients with gastritis and peptic ulceration. Lancet, 1:1.3101.314. 